\title{
Development of a Power Assist System of a Walking Chair Based on Human Arm Characteristics"
}

\author{
Yunfeng $\mathrm{WU}^{* *}$, Hitoshi NAKAMURA ${ }^{* * *}$, Yukio TAKEDA ${ }^{* *}$, \\ Masaru HIGUCHI ${ }^{* *}$ and Koichi SUGIMOTO ${ }^{* *}$ \\ **Department of Mechanical Sciences and Engineering, Tokyo Institute of Technology \\ 2-12-1 O-okayama Meguro-ku, Tokyo 152-8552, Japan \\ E-mail:wu.y.ab@m.titech.ac.jp, takeda.y.aa@m.titech.ac.jp \\ *** Toyota Motor Corporation \\ 1 Toyota-Cho, Toyota City, Aichi Prefecture 471-8571, Japan
}

\begin{abstract}
In this paper, design of control system and power combination mechanism of a power assist system of the walking chair was discussed based on kinetostatic characteristics of human arm. The walking chair is a welfare walking machine which is an alternative vehicle of the wheelchair, and expected to be driven by user's cranking operation with assisting actuator. To efficiently utilize user power as much as possible for long locomotion without giving much fatigue to the user while providing comfortable driving feeling to the user, the human arm characteristics were taken into consideration. Kinetostatic characteristics of the human arm were experimentally investigated for its modeling. This model was applied to the design of mechanism and control system of the power assist system of the walking chair, and design parameters were determined for achieving comfortable driving feeling and efficient utilization of user power.
\end{abstract}

Key words: Walking Chair, Power Assist System, Biomechanics, Man Machine System, Welfare Machine Engineering

\section{Introduction}

Japan has become a seriously aging society, and this problem will become even more serious in the near future. Many other developed countries also face the same problem. Therefore, development of technology to support the welfare of such a society is strongly required. For more than seventeen years, Funabashi, Takeda et al. have been involved in the development of a walking chair which is an alternative vehicle of the wheelchair ${ }^{(1),(2)}$. The walking chair has legs under its seat to enable a user who cannot walk to move freely on such terrains as horizontal planes, slopes and stairs including irregularities such as minute steps in our living space. As shown in Fig. 1(a), the walking chair is expected to be driven by user's cranking operation. To this end, a closed-loop four-bar mechanism with a single degree of freedom, fundamental mechanism, was adopted to generate a periodic leg motion on a horizontal floor. More recently, researches on walking robots for the elderly people and disabled persons were being intensively done. The WL-15 of Waseda University ${ }^{(3)}$ and Toyota Motor Corporation's personal robot i-foot ${ }^{(4)}$ have been developed. These walking robots are driven by autonomous control system, and are not powered by their users.

Currently, we are involved in the development of a power assist system for the walking chair ${ }^{(5)}$. The walking chair suffers from large variation in driving torque of the input shaft

${ }^{*}$ Received 20 Dec., 2006 (№. 06-0293) [DOI: 10.1299/jamdsm.1.141] 


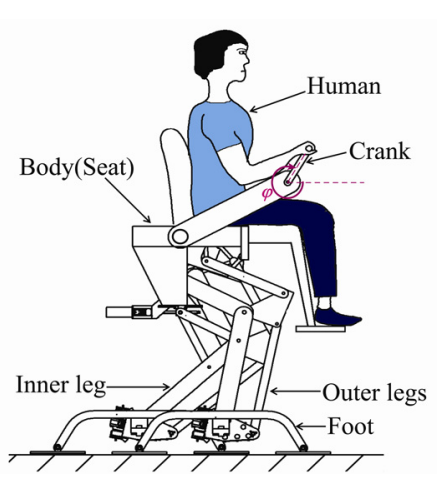

(a) Schematic view

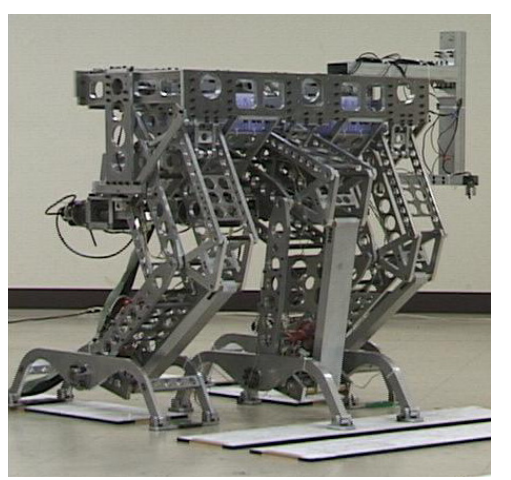

(b) Prototype

Fig.1 The walking chair

of the fundamental mechanism of the leg mechanism. This makes it difficult for a user to drive the walking chair without assistance. The power assist control system not only assist user's driving to against heavy load, but also utilizes user power effectively to save power of battery and provide appropriate driving feeling to the user. That is why we are studying a hybrid drive system as a power assist system that combines power of assisting actuator(s) and user power, in which the user power is given by rotating a crank through user's hand. For practical using of the walking chair, the power assist system must satisfy the following requirements.

(a) User intention must be reflected on the walking speed of the walking chair. To extract information corresponding to user intention from the signal/data of user's driving torque or velocity is a better way of making the system simple. In our study, we use velocity or torque signals generated by the user.

(b) User must be able to drive the walking chair comfortably even though the load torque of the walking chair greatly varies.

(c) The power assist system must efficiently utilize the user power and power of actuators.

In our previous work $^{(5)}$, we discussed the power combination mechanism and its control method for a periodic walk on a horizontal floor to achieve a stable output velocity and a smooth user's torque according to user intention. Then, a power assist system that satisfies with the requirement (a) was developed. In our present work, a power assist system that can satisfy the requirements (b) and (c) is investigated taking human arm characteristics into consideration.

To achieve requirements (b) and (c), the composition of the power assist system must be constructed based on not only the load torque characteristics of the walking chair but also the characteristics of user's cranking operation. In our work, a kinetostatic model of human arm which can be easily implemented was developed. Many researchers have modeled human body mechanism for the purpose of analyzing human's motion and developing welfare apparatus and sports wear, etc ${ }^{(6-10)}$. Oshima et al. proposed a model in which functionally separated muscles were introduced ${ }^{(9)}$. However, the model is restricted in the static condition. Obinata et al. proposed a human arm model including muscle characteristics, and the model was applied to a design of a hand rim of a wheelchair considering torque generated by human and workload ${ }^{(10)}$.

In the present paper, in order to realize a power assist system of the walking chair which satisfies above-mentioned three requirements, we first show the characteristics of the driving torque of the walking chair. Crank driving characteristics of the human arm were experimentally investigated to develop its kinetostatic model. In the model, several constraints were introduced considering the situation when a human drives a crank by the human arm sitting on a seat as well as physical properties such as maximum joint torque. The model's effectiveness was investigated through comparison with experimental results. 


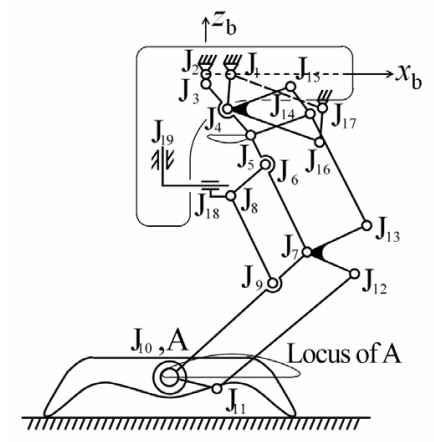

(a) Composition of the leg mechanism

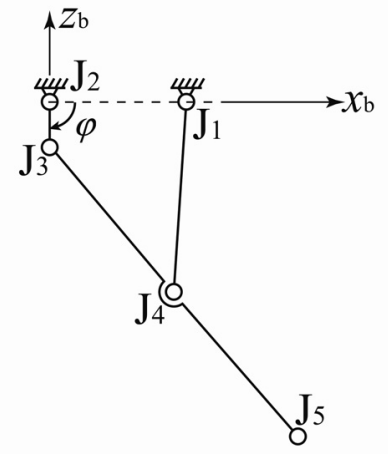

(b) Fundamental mechanism

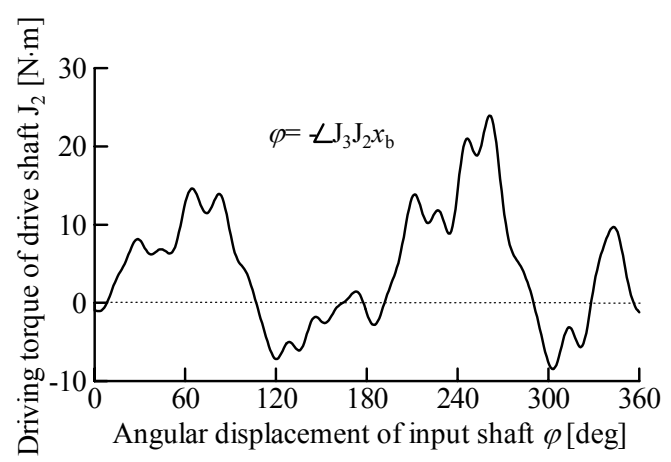

(c) Change of the driving torque of the walking chair (walking period : $T=4 \mathrm{~s}$ )

Fig. 2 Composition of the leg mechanism and change of driving torque

Table 1 Dimensions and masses of the walking chair used in experiments

\begin{tabular}{|l|l||l|l|}
\hline Height & $0.91 \mathrm{~m}$ & Mass & $88.1 \mathrm{~kg}$ \\
\hline Width & $0.76 \mathrm{~m}$ & Mass of inner leg & $19.8 \mathrm{~kg}$ \\
\hline Step length & $0.30 \mathrm{~m}$ & Mass of outer legs & $17.4 \mathrm{~kg}$ \\
\hline
\end{tabular}

Hardware and software composition of the power assist system are proposed based on the proposed human arm model and the characteristics of the driving torque of the walking chair. Influence of the design parameters of the power assist system on its performance such as output velocity deviation and power efficiency is investigated by simulations. Finally, a power assist system is constructed, and its effectiveness is confirmed by experiments.

\section{Composition of the Walking Chair and its Driving Torque}

Kinematic composition of the leg mechanism of the walking chair is shown in Fig. 2(a). Leg motion for a horizontal terrain (fundamental motion) is generated by rotating the input link $\mathrm{J}_{2} \mathrm{~J}_{3}$ at a constant velocity. A planar closed-loop four-bar approximate-straight-line mechanism $\left(\mathrm{J}_{1} \mathrm{~J}_{2} \mathrm{~J}_{3} \mathrm{~J}_{4} \mathrm{~J}_{5}\right)$ shown in Fig. 2(b) is used as fundamental mechanism to generate this motion. Adjusting motion with two degrees of freedom given at the joints $J_{18}$ and $J_{19}$ is combined with the fundamental motion to generate motions for slopes, large steps and stairs. Fundamental and adjusting motions are combined by a pantograph mechanism. The fundamental mechanism is designed as to be driven by both user and assisting actuator(s), while the adjusting mechanism is driven by actuators. When the walking chair walks on slopes, steps and stairs at a constant velocity, the driving torque of the fundamental mechanism is almost the same as that on a horizontal terrain. The dimensions and masses of the prototype walking chair (see Fig. 1(b)) are shown in Table 1. The driving torque of the drive shaft $\mathrm{J}_{2}$ respect to its angular displacement is presented in Fig. 2(c) in the case when the walking chair is walking on a horizontal plane with walking period $T=4 \mathrm{~s}$. As it can be seen in the figure that the driving torque sinusoidally changes (both positively and 


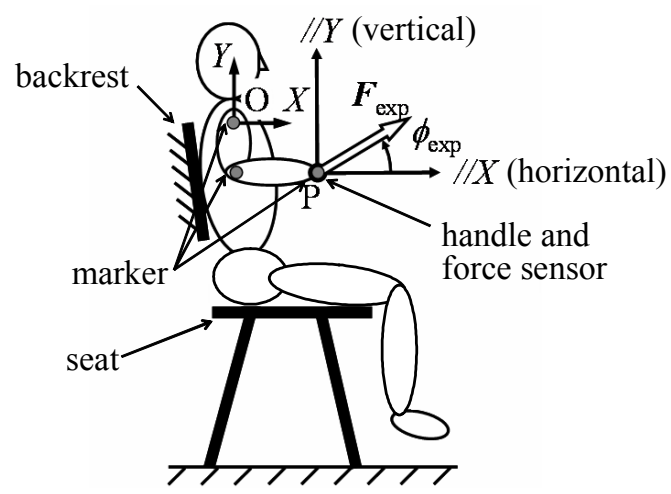

Fig. 3 View of experiments for investigation of kinetostatic characteristics of human arm

negatively) according to acceleration and deceleration of the foot while the drive shaft (input link $\mathrm{J}_{2} \mathrm{~J}_{3}$ ) rotates at a constant velocity. We use this torque as the input torque of the fundamental mechanism that should be obtained as the output torque of the power assist system that follows.

\section{Maximum Driving Torque of a Crank by Human}

\subsection{Measurement of the Maximum Driving Torque}

We conducted experiments to measure the magnitude of the maximum force $F_{\text {exp }}$ that can be applied to the fixed handle through a gripping hand when a human sits with his/her back leaning against the backrest and with his/her feet in the air as shown in Fig. 3.

The relationship between the maximum force $F_{\text {exp }}$ to its generating direction $\phi_{\exp }$ and the handle position $\mathrm{P}$ was investigated. The magnitude $F_{\text {exp }}$ and the direction $\phi_{\exp }$ were measured by a force sensor (FORCE-MOMENT SENSOR, by NITTA Corp.) attached between the handle and the base. Human arm configuration was measured using a 3D optical position sensor (OPTOTRACKR3020, by Northen Digital Inc.). Experimental results are summarized as follows:

(a) The magnitude of the force largely depends on its direction and the handle position.

(b) The arm configuration was changed according to the direction of the force utilizing the redundancy of human arm for the same handle position. This configuration change is done by moving the position of the shoulder joint's center.

(c) When the positions close to the workspace boundary where the muscles are stretched or shrank, the human arm can generate less force than expected.

(d) The maximum force $F_{\text {exp }}$ depends on the reaction from the seat and the backrest.

\subsection{The Kinetostatic Model of the Human Arm}

For the analysis of the maximum generating force at the hand, a kinetostatic model of human arm is proposed. In the model, to reflect the experimental result (b) in the previous subsection, that is, the shoulder joint's center is moved to an appropriate position where human arm can easily generate force to the specific direction, we proposed a kinetostatic model of the human arm as shown in Fig. 4. The proposed kinetostatic model have three prismatic joints $\left(\mathrm{P}_{1}, \mathrm{P}_{2}, \mathrm{P}_{3}\right)$ to move the shoulder joint's center, three revolute joints $\left(\mathrm{R}_{1}, \mathrm{R}_{2}\right.$, $R_{3}$ ) to obtain a spherical motion of the shoulder and a revolute joint $R_{4}$ at the elbow. Links 4 and 5 are zero mass and zero dimensions. The stationary coordinate system $\mathrm{O}-X Y Z$ is located so that the origin $\mathrm{O}$ coincides with the shoulder joint center when human does not generate any force and $X Y$ plane is the sagittal plane. The relationship between the joint torque $\tau=\left\{\tau_{1}, \tau_{2}, \tau_{3}, \tau_{4}\right\}^{\mathrm{T}}$ and generating force $F=\left\{F_{\mathrm{x}}, F_{\mathrm{y}}, F_{\mathrm{z}}\right\}^{\mathrm{T}}$ at the hand can be written as

$$
\tau=J^{T}(\theta) F+g(\theta)
$$

where $J(\theta)$ is the jacobian matrix, $\theta=\left\{\theta_{1}, \theta_{2}, \theta_{3}, \theta_{4}\right\}^{\mathrm{T}}$ is angular displacement of revolute 


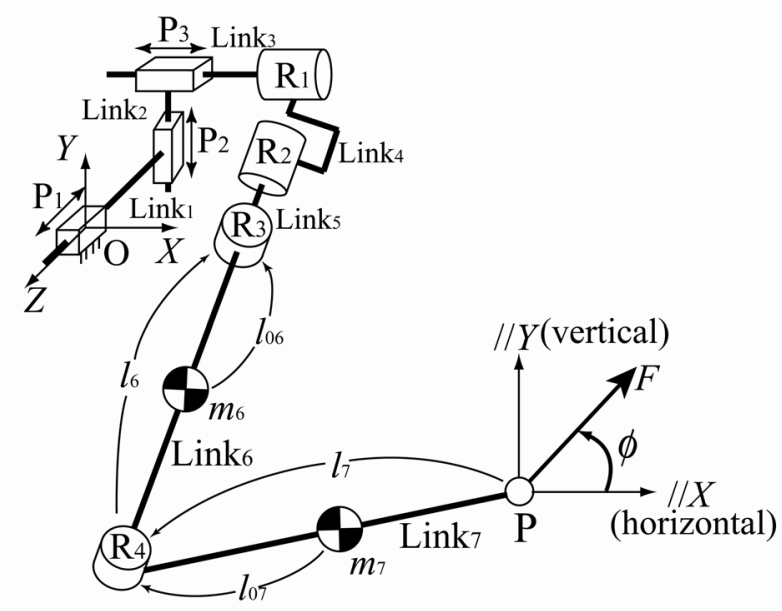

Fig. 4 Kinetostatic model of the human arm

joints $\mathrm{R}_{1} \sim \mathrm{R}_{4}$, and $g(\theta)$ is the gravitational term.

To reflect the kinetostatic characteristics of human arm obtained through experiments presented in the previous section, following constraint conditions are introduced to obtain the maximum generating force at the hand.

1) Maximum torque constraint:

For the torque in each revolute joint $\tau_{\mathrm{i}}$, the maximum torque constraint is introduced.

$$
-\tau_{\text {iemax }} \leq \tau_{i} \leq \tau_{\text {if } \max }
$$

Here, $\tau_{i f \max }$ and $\tau_{i e \max }$ are maximum generating torques in flexture and extention directions of $i$-th joint measured by experiments.

2) Generating torque ratio constraint:

Since muscles such as bi-articular muscles usually drive not only one joint, but several joints, we considered that when a joint's torque reaches its maximum, it is impossible for other joints' torque to reach their maximum. To reflect this, we introduce a constraint condition shown in Eq. (3). This condition restricts the generating torque ratio in the revolute joints with variable $n_{\mathrm{i}}$ as shown in Eq. (4).

$$
n_{i}=\left\{\begin{array}{c}
\sum_{i} n_{i}<2 \\
0\left(-k_{2} \tau_{i e \max } \leq \tau_{i} \leq k_{2} \tau_{i f \max }\right) \\
0.6\left(-k_{1} \tau_{i e \max } \leq \tau_{i} \leq-k_{2} \tau_{i e \max }, k_{2} \tau_{i f \max } \leq \tau_{i} \leq k_{1} \tau_{i f \max }\right) \\
1\left(\tau_{i}<-k_{1} \tau_{i e \max }, k_{1} \tau_{i f \max }<\tau_{i}\right)
\end{array}\right.
$$

In Eq. (4), $k_{1}$ and $k_{2}$ are functions of the handle position and generating force direction, and these functions were determined by trial and error operations for minimizing the error between the experimental results and the analytical results which are calculated using the proposed kinetostatic model.

3) Maximum reaction force constraint:

The maximum reaction forces from the seat and backrest are considered introducing the following constraint.

$$
F \leq F_{\text {re } \max }(\phi)
$$

Here, $F_{\text {remax }}(\phi)$ is the maximum reaction force given to the user considering the reaction from the seat and backrest which is measured by experiments.

Change of the shoulder joint center according to the force direction and the handle position is considered by constructing a database which relates the force direction $\phi$ and prismatic joints displacement. This database was obtained by experiments. The flowchart of 


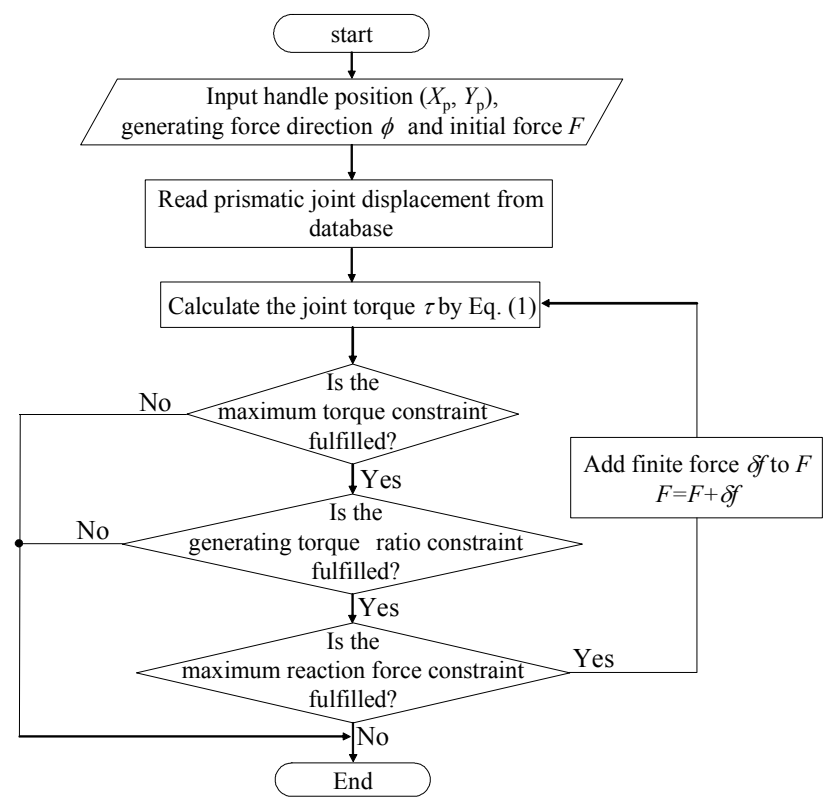

Fig. 5 Flowchart of the process for calculating the maximum generating force

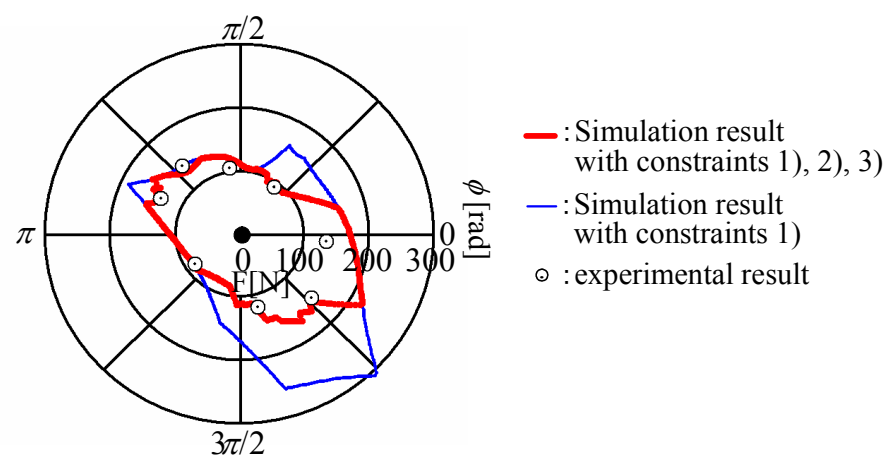

Fig. 6 Relationship between the maximum generating force and its direction obtained with simulation and experiment

Table 2 Mass and length properties of the subject's arm

\begin{tabular}{|c|c|c|c|c|c|}
\hline$m_{6}$ & $m_{7}$ & $l_{6}$ & $l_{7}$ & $l_{06}$ & $l_{07}$ \\
\hline $1.70 \mathrm{~kg}$ & $1.01 \mathrm{~kg}$ & $0.25 \mathrm{~m}$ & $0.35 \mathrm{~m}$ & $0.13 \mathrm{~m}$ & $0.13 \mathrm{~m}$ \\
\hline
\end{tabular}

total calculation process of the maximum generating force at the handle is shown in Fig. 5 . As shown in the figure, the maximum force is obtained through iterative computations.

\subsection{Validity of the Proposed Kinetostatic Model}

Both the simulation and the experimental results of the maximum generating force of the human arm at the handle with respect to the force direction are shown in Fig. 6. Mass and length properties of the subject used in the simulation are shown in Table 2. Each parameter was estimated based on mass ratios and center of mass location ratios in male Japanese athletes ${ }^{(11)}$. In both results, the position of handle is $\left(X_{\mathrm{p}}, Y_{\mathrm{p}}, Z_{\mathrm{p}}\right)=(0.32,-0.25$, $0.25)[\mathrm{m}]$. Figure 6 demonstrates that, the simulation results become closer to the experimental results in the case when the constraints 1) 3) are introduced than the case where only the constraint 1) is introduced. At other positions of handle, the simulation results almost coincide with the experimental results. These results show the validity of the proposed kinetostatic model of human arm for analyzing the maximum force generated by 


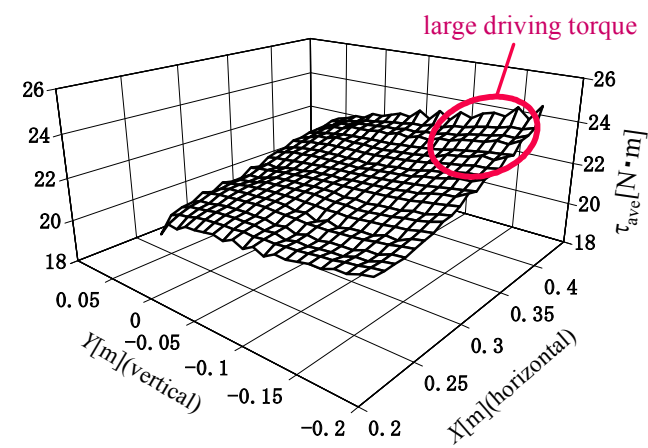

Fig. 7 Distribution of $\tau_{\text {ave }}$

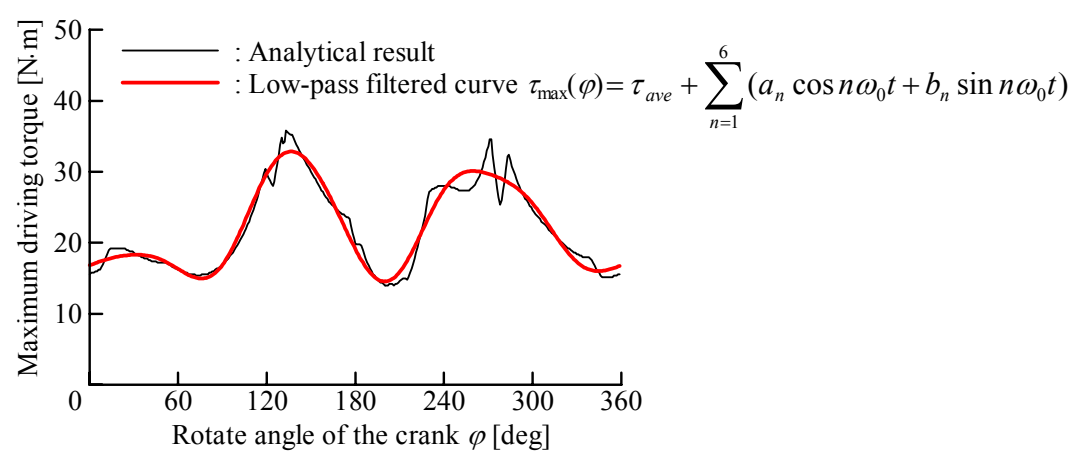

Fig. 8 The maximum generating torque by the human arm

the human.

\subsection{Simulation Result of the Maximum Driving Torque}

Optimal position of the crank center $\left(X_{\text {center }}, Y_{\text {center }}\right)$ was investigated using the proposed model. Average of the maximum driving torque $\tau_{\text {ave }}$ in one cycle of crank rotation was considered as the evaluation index. Here the maximum driving torque is obtained as multiplying the maximum generating force by the radius of crank. Distribution of $\tau_{\text {ave }}$ with the position of the crank center ( $\left.X_{\text {center }}, Y_{\text {center }}\right)$ obtained using the proposed kinetostatic model is shown in Fig. 7. From the result, the position of the crank center was determined as $(0.41,-0.16)[\mathrm{m}]$. Change of the maximum driving torque with respect to the crank angle $\varphi$ obtained with the proposed kinetostatic model is shown in Fig. 8. In Fig. 8, analytical result of the maximum driving torque is discrete value. From preliminary experiments, we found that human is not sensitive enough to feel the relatively high frequency and small magnitude of torque variation, and equation description of the maximum driving torque is better than discrete values in the control program. Therefore low-pass filtered curve using the Fourier transformation as shown in Fig. 8 was used as $\tau_{\max }(\varphi)$. We will use the low-passed curve as the maximum generating torque to drive a crank as a function of the angle $\varphi$, and it is denoted as $\tau_{\max }(\varphi)$. In the next section, we will design the power assist system of the walking chair based on the maximum torque $\tau_{\max }(\varphi)$ so as to supply comfortable driving feeling to the user and high power efficiency of the system.

\section{Composition of the Power Assist System}

To provide a comfortable driving feeling to the user and constant output velocity under heavily varying load torque, power combination mechanism of the power assist system is designed so that the torque ratio and velocity ratio between user's input and output to the input shaft of the leg mechanism of the walking chair can be arbitrarily adjusted ${ }^{(5)}$. Based 


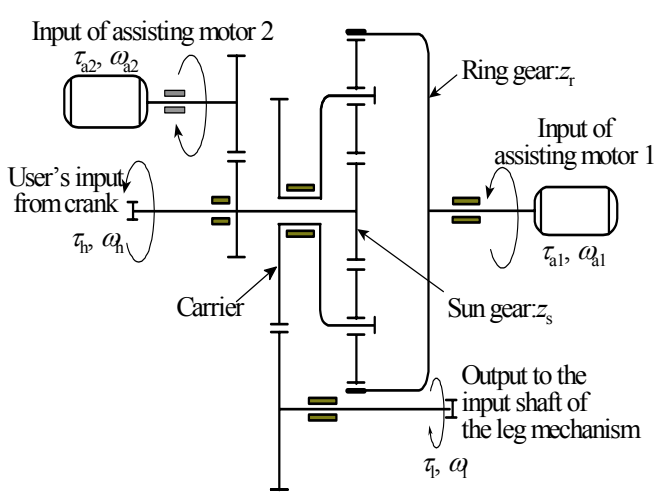

Fig. 9 Composition of the power combination mechanism of the power assist system

on the human arm characteristics, the control method of the power assist system is proposed to provide comfortable driving feeling, and design parameters of the system are clarified.

\subsection{Power Combination Mechanism}

In order to arbitrarily adjust both velocity ratio and torque ratio, the power combination mechanism of the power assist system was composed as shown in Fig. 9. In this power combination mechanism, a pair of spur gears is used to adjust the torque ratio, and a planetary gear train is used to adjust the velocity ratio. To adjust the ratios of velocity and torque, two motors are used. Since the most important thing for the user is freedom from load torque variation, the shaft for user input was connected to the shaft of assisting motor 2 by a pair of spur gears. And, because output velocity must be kept constant while reflecting user intention, the output shaft is connected to the shaft of assisting motor 1 by a planetary gear train. Both torque and velocity relationships are derived as Eqs. (6) and (7), where $N$ is the gear ratio between the ring gear and the sun gear in the planetary gear train. In the Eqs. (6) and (7), $\tau$ is the torque, $\omega$ is the angular velocity, subscripts $h$, a1, a2 and 1 mean the user, assisting motor 1 , assisting motor 2 and load, respectively.

$$
\begin{gathered}
\tau_{a 1}=\frac{N}{N+1} \tau_{h}, \tau_{h}+\tau_{a 2}=\frac{1}{N+1} \tau_{l} \\
\omega l=\frac{\omega_{h}+N \omega_{a 1}}{N+1}
\end{gathered}
$$

\subsection{Control Method}

1) Average velocity control method for assisting motor 1

We proposed in Ref. (5) average velocity control method in which the desired output velocity of the system is determined by the average velocity $\omega_{\mathrm{oh}}$ of user driving. $\omega_{\mathrm{oh}}$ is obtained from the user's velocity $\omega_{\mathrm{h}}$, by passing it through a low pass filter with a cut-off period $T_{\text {period. }}$. User intention is extracted from $\omega_{\mathrm{h}}$, and the influence of user's velocity variations on the output velocity is reduced by averaging. Specifically, the desired output velocity $\omega_{\mathrm{ol}}$ is obtained by

$$
\omega_{o l}=K \omega_{o h},
$$

where $K$ is a constant velocity ratio between input and output velocities. Substituting $\omega_{\text {oh }}$ and $\omega_{01}$ into $\omega_{1}$ and $\omega_{1}$ of Eq. (7), the desired velocity of assisting motor $\omega_{0 \mathrm{oa}}$ is obtained as

$$
\omega_{\mathrm{oal}}=\frac{K(N+1) \omega_{\mathrm{oh}}-\omega_{\mathrm{h}}}{N}
$$

2) Impedance control method for assisting motor 2

To provide comfortable driving feeling for the user, an impedance control method was applied to the assisting motor 2 assuming the following virtual inertia system 


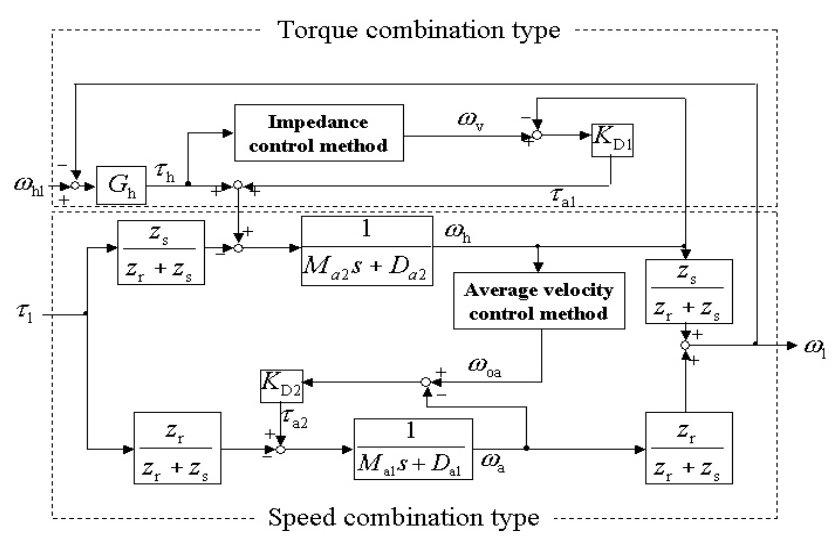

Fig. 10 Block diagram of the proposed power assist system

$$
\dot{\omega}_{\mathrm{v}}=\frac{1}{M_{\mathrm{v}}}\left(\tau_{\mathrm{h}}-D_{\mathrm{v}} \omega_{\mathrm{v}}\right),
$$

where $\omega_{\mathrm{v}}$ is the velocity of virtual system, and $M_{\mathrm{v}}$ and $D_{\mathrm{v}}$ are the virtual moment of inertia and the virtual damping coefficient ${ }^{(5)}$. Extracting user intention from user torque $\tau_{\mathrm{h}}$ measured by a torque sensor, $\dot{\omega}_{\mathrm{v}}$ is obtained in (10) and $\omega_{\mathrm{v}}$ is obtained by integrating $\dot{\omega}_{\mathrm{v}}$. Feedback torque $\tau_{\mathrm{a} 2}$ is given to the assisting motor 2 to let its velocity $\omega_{\mathrm{a} 2}$ follow the virtual velocity $\omega_{v}$. Use of such impedance control method makes the user feel as if he were driving a virtual inertia system without feeling torque variation included in the load. $M_{\mathrm{v}}$ and $D_{\mathrm{v}}$ are control parameters. $M_{\mathrm{v}}$ mainly determines the velocity variation of user's velocity, while $D_{\mathrm{v}}$ mainly determines the magnitude of user's driving torque.

For controlling user's driving torque according to the phase of the crank, the virtual damping coefficient $D_{\mathrm{v}}$ used in the impedance controller was taken as a design parameter. In order to reflect the maximum torque characteristics $\tau_{\max }(\varphi)$ shown in Fig. 8, the value of $D_{\mathrm{v}}$ was determined as a function of crank phase $\varphi$ as the following equation introducing an weight $A$ and desired average torque of user $\tau_{\text {have }}$

$$
D_{v}(\varphi)=\frac{\left[A \tau_{\text {hdev }} /\left(\tau_{\max }-\tau_{\text {ave }}\right)\right]\left[\tau_{\max }(\varphi)-\tau_{\text {ave }}\right]+\tau_{\text {have }} .}{\omega_{d h}} .
$$

Here, $\omega_{\mathrm{dh}}$ is the desired driving velocity of the user, $\tau_{\mathrm{ave}}$ is the average of the maximum driving torque, $\tau_{\max }$ is the maximum of $\tau_{\max }(\varphi)$ with respect to $\varphi$, and $\tau_{\text {hdev }}$ is the user's driving torque variation obtained from experiments. $\tau_{\text {hdev }}$ and $\tau_{\text {have }}$ are different by users. By changing the weight $A$, driving torque variations are obtained. In the same way, by changing the desired average driving torque $\tau_{\text {have }}$, variable magnitude of the torque are obtained. These are to be determined as universal design parameters for each user.

Figure 10 is a block diagram of the overall controller of the proposed power assist system. By using the proposed power assist system, we expect that a user can drive the crank at a velocity with natural variation and without feeling variations of load torque, which results in a constant velocity of the output shaft.

\subsection{Design Parameters}

As additional design parameters of the proposed power assist system, gear ratio $N$ of planetary gear train and phase shift $\psi$ of the torque curve of load torque relative to the human torque are considered, because they mainly affect the power efficiency of the power assist system.

Then, design parameters of the proposed power assist system are summarized as

(a) Cut off period of low pass filter $T_{\text {period: }} T_{\text {period }}$ is used to filter user's driving velocity.

Since the user's driving velocity varies periodically and the most main frequency component is $0.5 \mathrm{~Hz}$ in stable walking condition, it is determined as 2 second. 


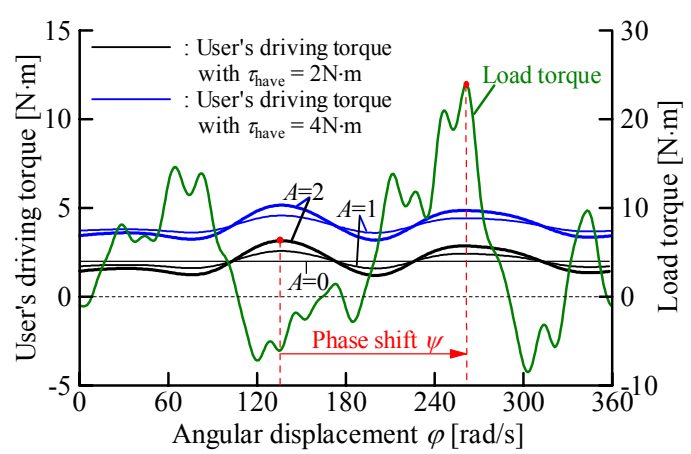

Fig. 11 Influence of parameters $A, \tau_{\text {have }}$, phase shift $\psi$

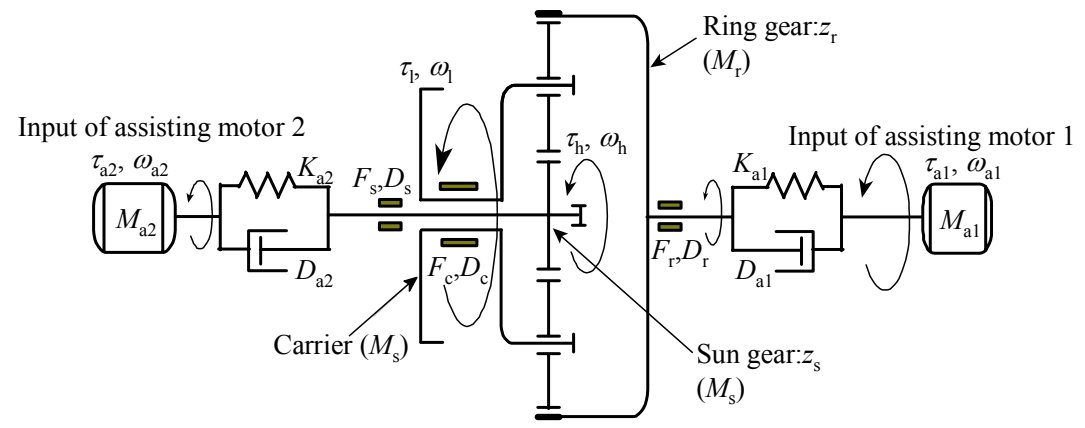

Fig. 12 Simulation model of the power assist system

Table 3 Parameters of power assisting system in Numerical simulation

\begin{tabular}{|c|c||c|c|}
\hline$F_{\mathrm{s}}$ & $0.2 \mathrm{~N} \cdot \mathrm{m}$ & $K_{\mathrm{a} 1}$ & $1000 \mathrm{~N} \cdot \mathrm{m} / \mathrm{rad}$ \\
\hline$D_{\mathrm{s}}$ & $0.2 \mathrm{~N} \cdot \mathrm{ms} / \mathrm{rad}$ & $D_{\mathrm{a} 1}$ & $0.1 \mathrm{~N} \cdot \mathrm{ms} / \mathrm{rad}$ \\
\hline$F_{\mathrm{r}}$ & $0.2 \mathrm{~N} \cdot \mathrm{m}$ & $K_{\mathrm{a} 2}$ & $1000 \mathrm{~N} \cdot \mathrm{m} / \mathrm{rad}$ \\
\hline$D_{\mathrm{r}}$ & $0.2 \mathrm{~N} \cdot \mathrm{ms} / \mathrm{rad}$ & $D_{\mathrm{a} 2}$ & $0.1 \mathrm{~N} \cdot \mathrm{ms} / \mathrm{rad}$ \\
\hline$z_{\mathrm{r}}$ & 72 & $M_{\mathrm{a} 1}$ & $0.1 \mathrm{kgm}$ \\
\hline$z_{\mathrm{s}}$ & 24 & $M_{\mathrm{a} 2}$ & $0.1 \mathrm{kgm}^{2}$ \\
\hline$M_{\mathrm{r}}$ & $0.2 \mathrm{kgm}^{2}$ & $M_{\mathrm{s}}$ & $0.1 \mathrm{kgm}^{2}$ \\
\hline
\end{tabular}

(b) Virtual damping coefficient $D_{\mathrm{v}}: D_{\mathrm{v}}$ is determined based on user's crank driving characteristics by Eq. (11) using supplementary parameters $A$ and $\tau_{\text {have }}$. And the change of the user's driving torque with respect to $A$ and $\tau_{\text {have }}$ is shown in Fig. 11.

(c) Virtual moment of inertia $M_{\mathrm{v}}: M_{\mathrm{v}}$ is determined by questionnaire.

(d) Gear ratio of planetary gear train $N$

(e) Phase shift of the torque curve of load torque relative to the human torque curve $\psi$ : the phase shift $\psi$ is defined as the difference between angular displacements of the maximum torque in user's driving torque and the load torque as shown Fig. 11.

In the next section, we show the simulation results on the influence of parameter $D_{\mathrm{v}}, N$ and $\psi$ to determine appropriate value of them.

\section{Determination of some Design Parameters through Simulations}

The simulation model is shown in Fig. 12, where connecting parts between assisting motors, sun gear, and ring gear are modeled with spring/damper $\left[K_{\mathrm{a} 1}, D_{\mathrm{a} 1}\right],\left[K_{\mathrm{a} 2}, D_{\mathrm{a} 2}\right]$, and connecting parts between each shaft and bearing are modeled with column friction $\left[F_{\mathrm{s}}, F_{\mathrm{c}}\right.$, $\left.F_{\mathrm{r}}\right]$ and viscous damping $\left[D_{\mathrm{s}}, D_{\mathrm{c}}, D_{\mathrm{r}}\right]$. Parameters used in the simulation are shown in Table 3.

5.1 Influence of the variation of human torque on the user's driving velocity deviation 


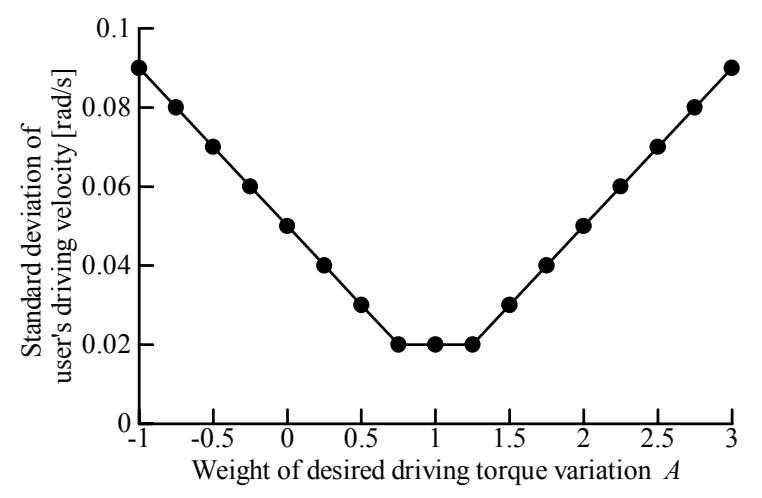

Fig. 13 Relationship between standard deviation of driving velocity and $A$

Prior to the simulation, difference of the user's maximum driving torque from the average driving torque $\tau_{\text {hdev }}$ was set as $1.16 \mathrm{~N} \cdot \mathrm{m}$ based on preliminary experiments, and desired driving velocity $\omega_{\mathrm{dh}}$ of user was set as $\pi \mathrm{rad} / \mathrm{s}$. Simulation results are shown in Fig. 13 , in which the relationship between the standard deviation of user's driving velocity and the weight $A$ is depicted. From the figure, the standard deviation of user's driving velocity becomes minimal when the desired driving torque variation in Eq. (11) is equal to or very close to user's driving torque characteristics. That is when $A$ is $[0.75,1.25]$ with $\tau_{\text {hdev }}$ $1.16 \mathrm{~N} \cdot \mathrm{m}$. And it is known from Fig. 13 that the standard deviation becomes larger as the desired driving torque variation separates from user's driving torque characteristics.

5.2 Influence of the gear ratio of the planetary gear train of the power combination mechanism and phase shift of the torque curve of load torque relative to the human torque on the power efficiency

Consider an ideal case where there is no friction torque and the user's crank driving velocity is kept constant. Then, the efficiency of the power assist system $\eta$ can be written as

$$
\eta=\frac{\int_{0}^{2 \pi}\left|\tau_{l}\left(\varphi_{l}\right)\right| d \varphi_{l} \times 100 \%}{\int_{0}^{2 \pi}\left|\tau_{h}\left(\varphi_{h}\right)\right| d \varphi_{h}+\int_{0}^{2 \pi} \frac{N}{N+1}\left|\tau_{l}\left(\varphi_{l}\right)\right| d \varphi_{l}+\int_{0}^{2 \pi}\left|\frac{\tau_{l}\left(\varphi_{l}\right)}{N+1}-\tau_{h}\left(\varphi_{l}\right)\right| d \varphi_{l}} .
$$

In Eq. (12), the numerator is constant. In the denominator, the first component is constant, and the second component is a function of $N$. Since the origin angle of drive shaft of the leg mechanism and the origin angle of the crank in the human input side don't coincide with each other, there exists a phase shift $\psi$. Therefore, the third component in the denominator of Eq. (12) is the function of $\psi$ as well as $N$. Therefore the equation Eq. (12) can be rewritten as a function of $\psi$ and $N$ as

$$
\eta=\eta(\psi, N)
$$

Relationship between efficiency $\eta$ and $(\psi, N)$ is shown in Fig. 14. Figure 14 demonstrates that efficiency $\eta$ increases according to the decrease of $N$, and that it changes according to the phase shift $\psi$. And it is also known that when $N$ is smaller than 0.5 , almost no change of efficiency $\eta$ observed with respect to $N$ to reach the boundary of the maximum efficiency. Actually, for the power combination mechanism, we use the average velocity control method to adjust the velocity ratio between human's driving velocity and output velocity, and $\psi$ always changes according to operation of the assisting motor 2 . Therefore it is not a good method to improve the efficiency $\eta$ by setting the phase shift $\psi$ as a constant value. As discussed above, by changing the gear ratio $N$ ( 8 to $1 / 3)$, the efficiency is significantly improved. From Fig. 14, it is known that $N$ is preferable to be less than $1 / 2$. On the other hand, if $N$ is too small, the sun gear of the planetary gear train becomes too small, 


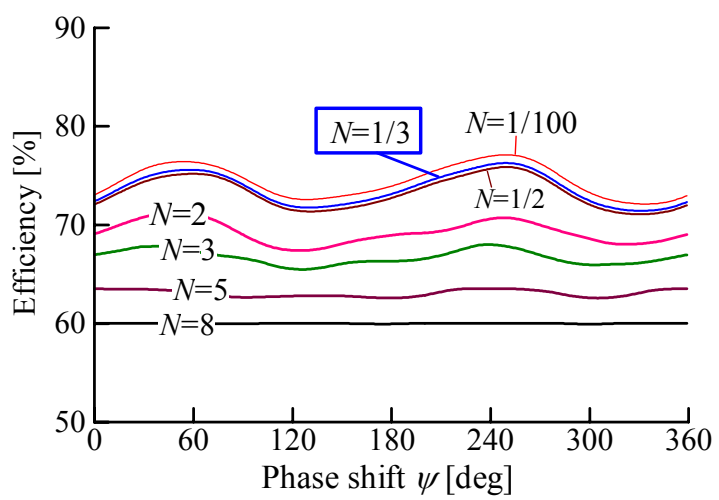

Fig. 14 Change of efficiency with respect to gear ratio $N$ and phase shift $\psi$

Table 4 Power relationship of different gear ratio $N$ obtained from simulations

\begin{tabular}{|c|c|c|c|}
\hline \multicolumn{2}{|c|}{$N$} & 3 & $1 / 3$ \\
\hline \multirow{3}{*}{ Power } & Human & $9.4 \mathrm{~W}$ & $9.4 \mathrm{~W}$ \\
\cline { 2 - 4 } & Assisting motor 1 & $18.6 \mathrm{~W}$ & $7.4 \mathrm{~W}$ \\
\cline { 2 - 4 } & Assisting motor 2 & $6.0 \mathrm{~W}$ & $15.6 \mathrm{~W}$ \\
\cline { 2 - 4 } & Load & $22.0 \mathrm{~W}$ & $22.0 \mathrm{~W}$ \\
\hline \multicolumn{2}{|c|}{ Efficiency } & $64.7 \%$ & $68.2 \%$ \\
\hline
\end{tabular}

and the stiffness of the total system will be decreased. Then, we determined the optimal gear ratio $N$ of the planetary gear train as $1 / 3$.

We investigated the power relationship and the efficiency of the power assist system through simulations for cases with different gear ratio $N$. Simulation results for cases of $N=3$ and $1 / 3$ are shown in Table 4 . From Table 4, it is known that the power efficiency is improved by changing $N$ from 3 to $1 / 3$ while the same user's driving power and load power are used.

\section{Experiments}

Experiments were conducted for the purpose of investigating the effectiveness of the proposed power assist system. The composition of the experimental apparatus of the power assist system is shown in Fig. 15. In experiments, the loading motor (200W, AC servomotor, Yasukawa Electric Corp.) generated the simulated driving torque of the walking chair shown in Fig. 2(c). In the experiment, we asked the user to drive the crank so as to follow a desired velocity $\pi \mathrm{rad} / \mathrm{s}$, while watching a speedometer for visual feedback. By this experimental condition, we expected that user's intention was to keep the driving velocity at $\pi \mathrm{rad} / \mathrm{s}$. The driving torque was measured by a torque sensor (TD020, by KUBOTA Corp). Assisting motor 1 (250W, DC servomotor, by MAXON Corp.) and assisting motor 2 (80W, DC servomotor, by MAXON Corp.) assisted user's crank driving based on the proposed control method.

Figure 16 shows an experimental result. Figure 16(a) shows that the output velocity followed well to the desired driving velocity $\pi \mathrm{rad} / \mathrm{s}$ with average of 3.16rad/s and standard deviation of $0.11 \mathrm{rad} / \mathrm{s}$, while the velocity variation of user driving is relatively large with average of $3.18 \mathrm{rad} / \mathrm{s}$ and standard deviation of $0.30 \mathrm{rad} / \mathrm{s}$. This means that use of the low pass filtered velocity of user's driving is effective to extract user's intention. Fig. 16(b) shows that the torque variation of user driving is small while load torque whose variation is large was generated from loading motor. Experimental result demonstrates that the proposed power assist system for the walking chair properly works in concert with user velocity and reflects the user intention, and human's driving torque is controlled well according to 


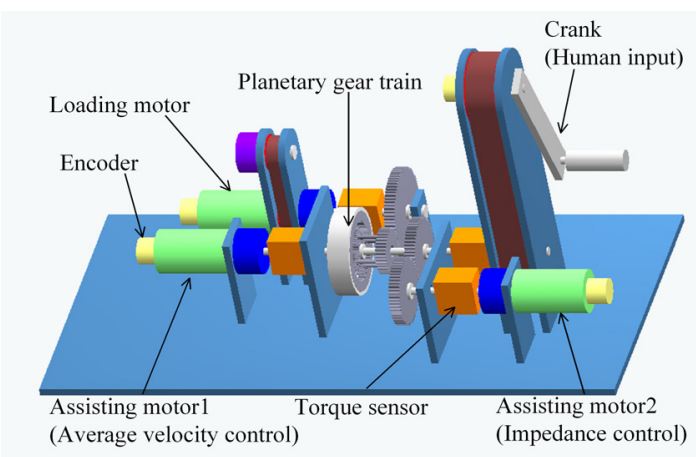

Fig. 15 Composition of the experimental apparatus of the proposed power assist system

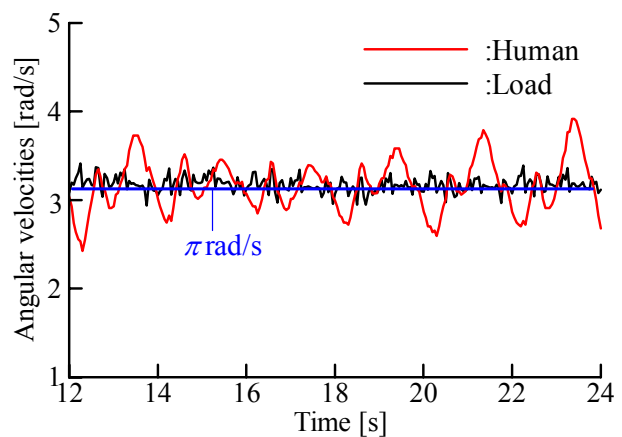

(a) Angular velocities

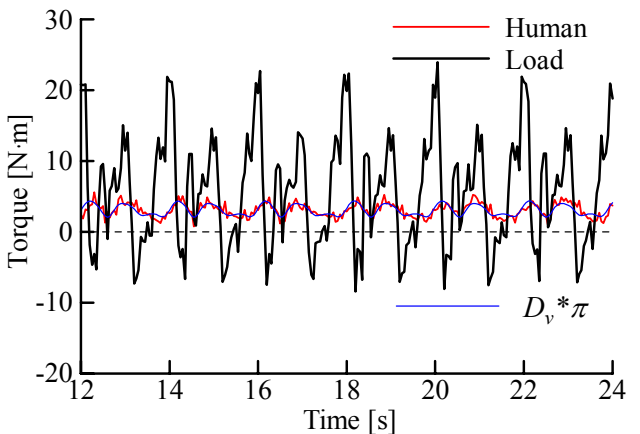

(b) Torques

Fig. 16 Change of angular velocities and torques when the proposed power assist system was used

Table 5 Results of intuitive evaluations by subjects (1: the worst, 5 : the best)

\begin{tabular}{|l|c|c|c|c|}
\hline & \multicolumn{4}{|c|}{ Weight $A$} \\
\cline { 2 - 5 } & 0 & 0.5 & 1.0 & 1.5 \\
\hline Evaluation & 3.3 & 3.0 & 4.2 & 3.8 \\
\hline
\end{tabular}

impedance control method.

We conducted experiments for different driving torque variation patterns by changing the control parameter $A$ which is varied in $[0,0.5,1,1.5]$. The crank driving feelings in each $A$ were intuitively evaluated by ten male subjects, aged between 22 to 27 years old, evaluations are given in five level [1 5], where 5 corresponds to the best driving feeling. The evaluation results by all subjects are summarized in Table 5. It is known from the table that subject's driving feeling becomes better when the weight $A$ equals to 1 that is the case where the driving torque curve is the same as the maximum generating torque by human. Therefore, by changing the driving torque variation according to the kinetostatic characteristics of user's cranking operation, better driving feeling can be supplied with the user.

\section{Conclusions}

In the present paper, a kinetostatic model of human arm was proposed to characterize human's cranking operation. Based on the kinetostatic characteristics of human's cranking operation and driving torque characteristics of walking chair, power combination mechanism and control system of the power assist system were constructed and their design parameters were clarified. Parameters were determined for achieving comfortable driving feeling and efficient utilization of human power. Simulation results showed that high efficiency can be archived by properly determining the values of design parameters. Also experimental results showed that user's comfortable driving feeling can be made better with consideration of the maximum driving torque by the human arm. 


\section{References}

(1) Funabashi, H., et al., Development of a walking chair with a self-attitude-adjusting mechanism for stable walking on uneven terrain, Proceeding of Tenth World Congress on the Theory of Machines and Mechanisms, (1999-6), pp.1164-1169.

(2) Takeda, Y., et al., Development of a walking chair (Fundamental investigation for realizing a practical walking chair), Proceedings of $4^{\text {th }}$ International Conference on Climbing and Walking Robots, (2001-9), pp.1037-1044.

(3) Sugahara, Y., et al., Support torque reduction mechanism for biped locomotor with parallel mechanisms, 2004 IEEE/RSJ International Conference on Intelligent Robots and Systems, (2004-10), pp.3213-3218.

(4) http://www.toyota.co.jp/en/special/robot/index.html, March 11 ${ }^{\text {th }}, 2004$.

(5) Wu, Y., et al., Development of a power assisting system of a walking chair, 2004 IEEE/RSJ International Conference on Intelligent Robots and Systems, (2004-10), pp.3207-3212.

(6) Hase, K., et al., Computer simulation study of human locomotion with a three-dimensional entire-body neuro-musculo-skeletal model (IV. Simulation of running motion and its transition process), JSME International Journal, Series C: Mechanical Systems, Machine Elements and Manufacturing, Vol. 45, No. 4(2002), pp.1065-1072.

(7) Nakamura, Y., et al., Somatosensory computation for man-machine interface from motioncapture data and musculoskeletal human model, IEEE Transaction on Robotics, Vol.21, No.1(2005), pp.58-66.

(8) Huynh, P., et al., Determination of human energy generated by skeletal muscle, Proceedings of IEEE International Workshop on Robot and Human Communication, (1997), pp. 272-277.

(9) Oshima, T., et al., Robotic analyses of output force distribution developed by human limbs, Proceedings of the 2000 IEEE International Workshop on Robot and Human Interactive Communication, (2000), pp.229-234.

(10) Sasaki, M., et al., Mobility evaluation of upper limbs in the operational space on wheelchair (in Japanese), The 2nd JSME Symposium on Welfare Engineering, No.02-34(2002), pp.205-208.

(11) Ae, M., et al., Estimation of inertia properties of the body segments in Japanese athletes (in Japanese), Biomechanisms, Vo.11(1992), pp.23-33. 